\title{
Perspectiva de ‘Bem-Estar Único’ em Sistemas Agroflorestais - uma Análise Bibliométrica
}

\author{
Perspective of 'One Welfare' in Agroforestry Systems - a Bibliometric Analysis
}

\author{
Anathan Bichel ${ }^{\mathrm{a}}$; Ediane Zanin*b \\ ${ }^{a}$ Universidade Estadual de Londrina, Programa de Pós-Graduação Stricto Sensu em Agronomia. PR, Brasil. \\ bUniversidade Estadual de Londrina, Programa de Pós-Graduação Stricto Sensu em Ciência Animal. PR, Brasil. \\ *E-mail ediane.z@hotmail.com
}

\begin{abstract}
Resumo
Os sistemas agroflorestais são adotados como forma de atender à produção sustentável. Este sistema de produção apresenta uma perspectiva de promoção do bem-estar único (One Welfare - OW). Com o objetivo de visualizar características e tendências para atender às premissas de OW em sistemas agroflorestais e desenvolver uma estrutura conceitual, foi realizada uma análise bibliométrica na literatura científica. Os dados foram extraídos da Web of Science (WoS) por meio da seleção de campos de pesquisa específicos para sistemas agroflorestais. Como resultado, 2.479 artigos foram encontrados e analisados no software VOSviewer, e um mapa bibliométrico foi produzido. Os principais clusters e interconexões foram descritos e discutidos. Os 2.479 artigos abrangeram a maioria das áreas de pesquisa, como agricultura, silvicultura, ecologia de ciências ambientais, ciências ambientais e vegetais, tecnologia; ou seja, artigos que integraram agrossilvicultura, produção animal, solo-planta e serviços ambientais; e, em menor número de publicações, biomedicina, ciências da vida, geografia física e farmacologia. Quatro grandes clusters foram formados por meio de interconexões: "crescimento e produtividade de plantas em sistemas agroflorestais", "biodiversidade, gestão e serviços ecossistêmicos", "dinâmica do carbono, biomassa e nitrogênio no solo" e "potencial da agrofloresta para segurança alimentar, mudanças climáticas, uso do solo e a sustentabilidade". Nossos resultados demonstraram que há um crescimento de artigos sobre sistemas agroflorestais, mas poucas pesquisas que abordaram diretamente o OW nesses sistemas. Há um grande potencial de progresso no tema abordado, dadas as interconexões formadas.
\end{abstract}

Palavras-chave: Bem-Estar. Clusters. Integração. Sustentabilidade.

\begin{abstract}
Agroforestry systems adopted as a way to meet the sustainable production. This production system presents a perspective for the promotion of One Welfare $(\mathrm{OW})$. In order to visualize characteristics and trends to meet the premises of $O W$ in agroforestry systems and develop a conceptual structure, a bibliometric analysis was carried out in the scientific literature. The data were extracted from the Web of Science (WoS) through the selection of specific research fields for agroforestry systems. As a result, 2.479 articles were found and analyzed using the VOSviewer software, and a bibliometric map was produced. The main clusters and interconnections have been described and discussed. The 2.479 articles covered most areas of research, such as agriculture, forestry, ecology of environmental sciences, environmental and plant sciences, technology; that is, articles that integrated agroforestry-animal production, soil-plant and environmental services; and, in a smaller number of publications, biomedicine, life sciences, physical geography and pharmacology. Four large clusters were formed through interconnections: "plant growth and productivity in agroforestry systems", "biodiversity, management and ecosystem services", "carbon dynamics, biomass and nitrogen in the soil" and "agroforestry potential for food security, climate change, land use and sustainability". Our results have shown that there a growth in articles on agroforestry systems, but little research that directly addressed $\mathrm{OW}$ in these systems. There is great potential for progress in the topic addressed given the interconnections formed.
\end{abstract}

Keywords: Welfare. Clusters. Integration. Sustainability.

\section{Introdução}

Sistemas agroflorestais têm sido empregados como estratégias para atender a produção de alimentos, geração de energia e uso da terra de forma mais sustentável (GEORGE et al., 2012). A combinação de atividades agrícolas, pecuárias e/ou florestais é traduzida em maior retorno econômico, impactos positivos em condições edáficas, microclimáticas e favorecimento ao sequestro anual de carbono (MBOW et al., 2014; ZOMER et al., 2016; TORRES et al., 2017; CÓRDOVA et al., 2019).
Além de todos esses aspectos socioeconômicos e ambientais já estudados, buscamos investigar perspectivas de sistemas agroflorestais como um meio direto e indireto de atender o bem-estar único. O bem-estar único tem como premissas melhorar o bem-estar animal, humano e os componentes ambientais; a fim de atender as metas mundiais de desenvolvimento sustentável (GARCÍA PINILLOS et al., 2016). A união destes fatores poderia promover a segurança alimentar, sustentabilidade, redução do sofrimento animal e humano e aumentar a produtividade de sistemas agrícolas e florestais (TARAZONA et al., 2020) - premissas similares a 
que se busca em sistemas agroflorestais (NAIR, 2011).

O bem-estar único (One Welfare, na língua inglesa) pode abranger várias áreas, complementar, sobrepor ou ser sobreposto as outras estruturas como a "One Earth, Planetary Health" (ROSENSTOCK et al., 2019), "One Helth" (HUMBOLDT-DACHROEDEN et al., 2020; TARAZONA et al., 2020), "Eco Health" (RAPPORT et al., 1998), "One Biology" (TARAZONA et al., 2020) e "One Stress" (BROOM; JOHNSON, 2019). A abordagem de "One Health" $(\mathrm{OH})$ que une saúde animal, saúde humana e ecossistemas (HUMBOLDT-DACHROEDEN et al., 2020); e a abordagem "Eco Health" (EH) definida por Rapport et al. (1998) como estável e sustentável, mantendo sua organização, autonomia ao longo do tempo e resiliência ao estresse; Costanza (1992) resumiu Eco Health como: saúde com homeostase; saúde com ausência de doença; saúde com diversidade ou complexidade; saúde com estabilidade ou resiliência; saúde com vigor ou espaço para crescimento; saúde com equilíbrio entre os componentes do sistema; já Rapport et al. (1998) citou a condição humana que é muito importante para manter um ecossistema sustentável dominado pelo homem e vice e versa. O conceito de bem-estar está se tornando aceito ainda como "One Biology", uma vez que implica que os princípios biológicos são exatamente o mesmo para humanos e todos os outros animais, mesmo com diferenças específicas entre as espécies e entre indivíduos. Investigar a biologia significa investigar humanos junto com todas as outras espécies (TARAZONA et al., 2020). E ainda para Broom e Johnson (2019) o uso dos termos estresse e bem-estar pela derivação de definições, estão relacionadas ao funcionamento e eficácia dos sistemas biológicos que os animais usam para regular suas vidas e lidar com as dificuldades.

$\mathrm{O}$ avanço sob uma perspectiva mais abrangente na interface humano-animal-ambiente depende de estudos e conhecimento multidisciplinares (HUMBOLDT-DACHROEDEN et al., 2020). Assim, este artigo utilizou a análise bibliométrica para explorar sistemas agroflorestais descritos na literatura acadêmica, e desta forma, visualizar as características e tendências para atender as premissas de bem-estar único, uma vez que, os índices possibilitam obter uma visão geral das características, dinâmicas, pontos fortes e deficiências (HUMBOLDT-DACHROEDEN et al., 2020). Os resultados foram utilizados para o desenvolvimento de uma estrutura conceitual de bem-estar único em sistemas agroflorestais.

\section{Desenvolvimento}

\subsection{Metodologia}

A pesquisa para esta revisão teve enfoque nos principais aspectos envolvendo sistemas agroflorestais e sua relação com o bem-estar único. Alguns autores abordaram apenas o termo sistemas agroflorestais para uso científico, sob diversos componentes, arranjos, dimensões no tempo e no espaço (NAIR, 1993); outros autores abordaram sistemas integrados de produção agropecuária (SIPA) e sistemas agroflorestais em grupos distintos (CARVALHO et al., 2014). Desta forma, por se tratar de uma pesquisa bibliográfica ampla e sob diversidade de termos e aplicações, utilizamos como meio de estudo avançado o tema "agroforestry system" ("sistemas agroflorestais" na língua portuguesa).

Os dados foram extraídos da Web of Science (WoS) devido à sua abrangência em relação ao assunto, estudos de alta qualidade e disponibilidade de dados acadêmicos multidisciplinares (DE SOUZA et al., 2019). Na WoS, para a pesquisa, foi utilizado o campo denominado "todos os anos", ou seja, até a data que pode ser acessado facilmente e com informações relevantes (DE SOUZA et al., 2019). A data e horário de levantamento de informações até o dia 11 de agosto de 2020. O campo "pesquisa avançada" foi acessado; e selecionado dados com base em títulos, resumo e palavraschave (DE SOUZA et al., 2019); operador booleano "OR"; restringindo os resultados pela língua "inglês" e documento tipo "artigo". Utilizou-se do caractere * para reunir de zero a vários caracteres, aspas (") para identificar exatamente a palavra/frase. O uso de apenas o termo agroforest* não foi pesquisado, devido a diversidade de aplicações, a exemplo: resíduos agroflorestais, que podem estar relacionados a potencial técnico de bioenergia; e não envolver o sistema agroflorestal (THORENZ et al., 2018).

Da busca no banco de dados da WoS, 2.479 artigos foram encontrados (material suplementar). As publicações foram então exportadas para o software VOSviewer versão 1.6.15. No software VOSviewer, mapas bibliométricos foram criados usando o banco de dados exportado anterior da WoS. A teia dos campos da ciência dos quais os termos foram extraídos foram as "palavras-chave", e a análise do tipo co-ocorrência foi selecionada com um número mínimo de 15 ocorrências de um termo.

Para mensurar a semelhança das palavras-chave com base no número de vezes que elas ocorreram juntas em diferentes artigos, foi utilizada a análise de co-ocorrência, a qual fornece informações sobre as principais outras palavraschave vinculadas a sistemas agroflorestais, e, portanto, pode ser usada para avaliar a estrutura de conhecimento do campo (HUMBOLDT-DACHROEDEN et al., 2020). Dessa forma, por meio das palavras-chave apresentadas, foi gerada a Figura 1 , que representa o potencial dos sistemas agroflorestais. 
Figura 1 - Mapeamento combinado das palavras-chave do autor citadas com mais frequência (a) e sobreposição, relacionadas a coocorrência e pontuações médias de publicação por ano (b) que surgiu na área de sistemas agroflorestais usando o banco de dados da WoS

a)

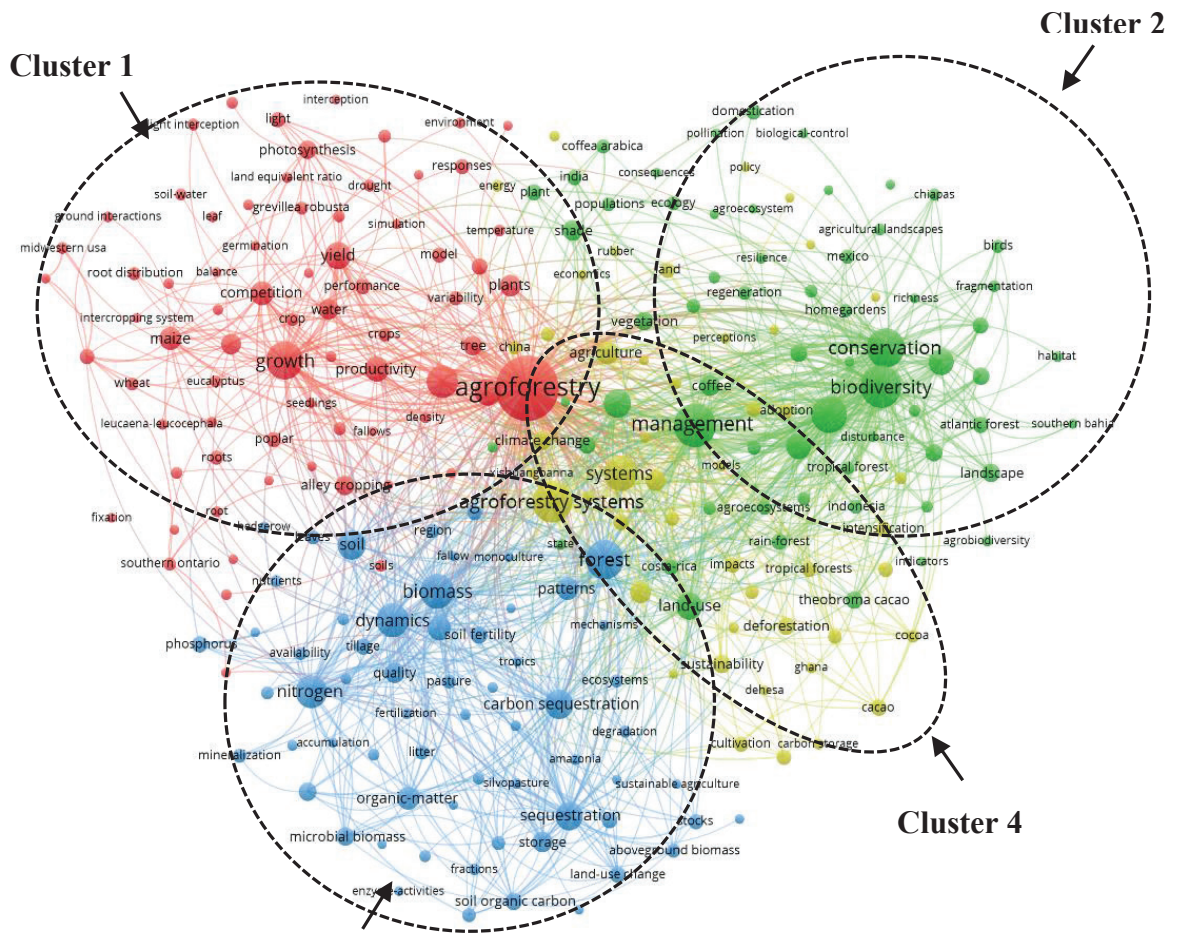

b)

\section{Cluster 3}

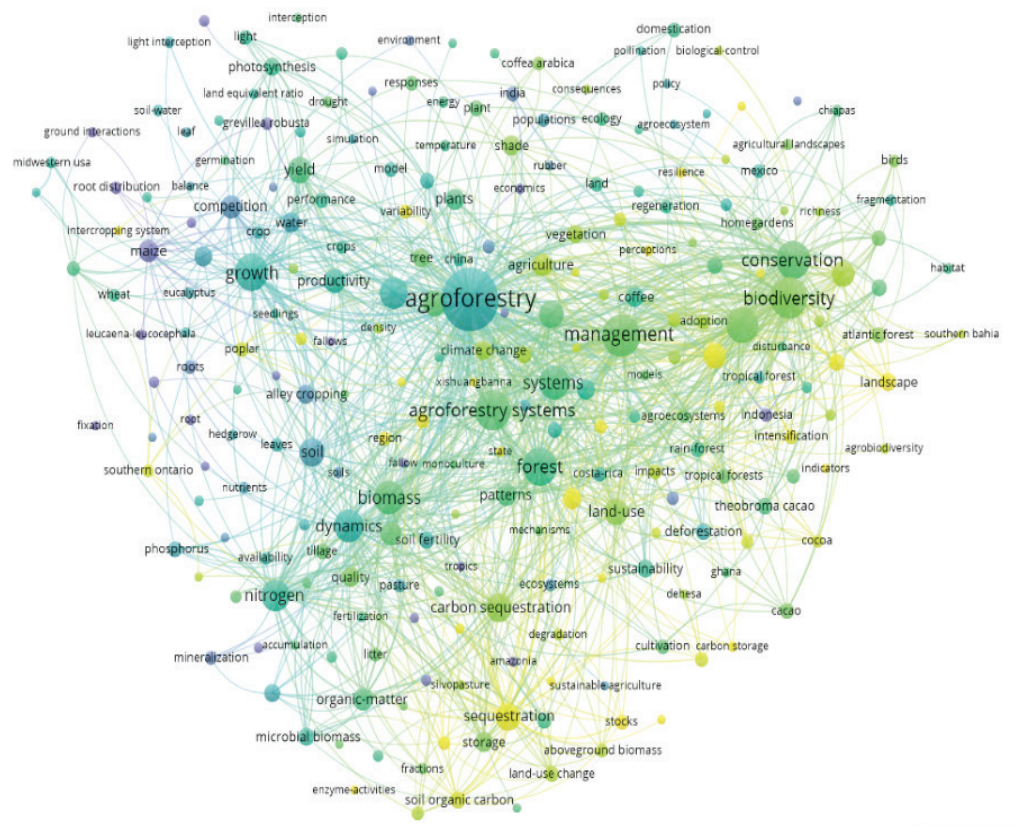

A vosviewer

Fonte: Dados da pesquisa.

Com o mapeamento bibliométrico e durante o desenvolvimento da escrita, os principais clusters de aplicação e interconexões foram descritos e discutidos. Por fim, a opinião crítica dos autores desta revisão foi sintetizada para facilitar o entendimento do assunto em geral, fornece uma perspectiva, análise crítica sob sistemas agroflorestais, bem como possíveis características e tendências para o bem-estar único.

\subsection{Interconexões em sistemas agroflorestais e o potencial para o bem-estar único}

O tema dos sistemas agroflorestais tem ganhado força na 
comunidade científica nos últimos anos. Foram encontramos 2.479 artigos no WoS, que integraram periódicos como Agroforestry Systems (691 articles), Agriculture Ecosystems Environmental (124), Forest Ecology and Management (76), Plant and Soil (43), Range Management and Agroforestry (28), Forests (26), Biodiversity and Conservation (25) e outros com menos de 25 publicações. As áreas de pesquisa envolvem, sobretudo agricultura, silvicultura, ecologia de ciências ambientais e vegetais, tecnologia, conservação da biodiversidade; e, em menor quantidade, biomedicina em ciências da vida, geografia física, farmacologia, ciências do comportamento, doenças infecciosas, parasitologia e outros.

Pesquisas relacionadas a humano-animal-ambiente dentro de sistemas agroflorestais são pouco investigadas (ROSENSTOCK et al., 2019). No entanto, no cenário atual, os impactos ocasionados pela forma incorreta de exploração e a desintegração de sistemas agrícolas e florestais tem gerado insegurança à vida dos seres vivos (TARAZONA et al., 2020). Consequências relacionadas as mudanças climáticas, poluição ambiental, desmatamento, perda de biodiversidade em terras de cultivo com práticas agrícolas inadequadas (ZERMEÑOHERNÁNDEZ et al., 2016), e doenças advindas de manejos incorretos ou perturbações no ecossistema (CATENACCI et al., 2018), levaram a um aumento no número de pesquisas sobre sistemas agroflorestais $(62.6 \%$ das publicações sobre sistemas agroflorestais estão no período 2010-2020, $\mathrm{n}=$ 2.479); em razão do potencial destes sistemas (LASCO et al., 2014).

Existem muitas iniciativas para mudar o uso de sistemas tradicionais de produção para alternativas mais "verdes" e com melhor utilização de recursos mundiais. A demanda humana por proteína animal, animais de trabalho e companhia, excederam a taxa de transformação desses sistemas mais integrados à sustentabilidade (ZERMEÑO-HERNÁNDEZ et al., 2016; BROOM, 2019). Essa demanda de consumo, impulsionou a criação de leis, códigos de práticas e políticas públicas para melhorias do bem-estar animal (LUNDMARK et al., 2014). O bem-estar animal foi aceito como uma questão fundamental pela Food and Agriculture Organization (FAO), Strategic Plan the World Organisation for Animal Health (OIE) e pela World Trade Organization (WTO), além de ser integrante dos critérios de sustentabilidade dos sistemas de produção animal (THIERMANN; BABCOCK, 2005). Atualmente, o conceito que integra as relações de bem-estar entre animal, humano e ambiente é o bem-estar único, o qual reconhece essas interconexões e fomenta a colaboração interdisciplinar mundialmente (GARCÍA-PINILLOS et al., 2016). A exemplo, a implementação da agenda 2030 da Organização das Nações Unidas (ONU), com plano de ação que indica 17 objetivos de desenvolvimento sustentável (ODS) e metas para apoiar e estimular ações de importância crucial para humanidade.

Em nosso estudo observamos estas interconexões e a interdisciplinariedade (Figura 1a e 1b). Quatro clusters
(Figura 1a) foram formados e possuem campos de maior frequência (DING; YANG, 2020) para agrofloresta, sistemas, biodiversidade, crescimento, produtividade, biomassa e sequestro de carbono; e estes possuem fortes interconexões com outros campos da ciência que apareceram com menor frequência. A rede de co-ocorrência formada a partir destes campos direcionam para estudos de "crescimento e produtividade de plantas em sistemas agroflorestais", "biodiversidade, gestão e serviços ecossistêmicos", "dinâmica do carbono, biomassa e nitrogênio no solo", e "potencial da agrofloresta para segurança alimentar, mudanças climáticas, uso do solo e a sustentabilidade".

$\mathrm{Na}$ Figura 1 a, o cluster 1 é sobre o "crescimento e produtividade de plantas em sistemas agroflorestais", possui 73 itens formadores, e envolveu as interações ecológicas, fisiológicas e agronômicas de plantas em sistemas agroflorestais. O cluster 2, envolveu "biodiversidade, gestão e serviços ecossistêmicos", possuiu 63 itens, com estudos de composição, diversidade e função de sistemas agroflorestais para com o ecossistema e o meio ambiente. $\mathrm{O}$ cluster 3 com "dinâmica do carbono, biomassa e nitrogênio no solo", possuiu 63 itens e envolveu estudos das mudanças no conteúdo e estoques de carbono e nitrogênio; formas de manejo e uso do solo, bem como as implicações sobre as mudanças climáticas. O cluster 4 , englobou o "potencial da agrofloresta para segurança alimentar, mudanças climáticas, uso do solo e a sustentabilidade", com 47 itens.

Os campos da ciência para a agrofloresta pareceram ter uma dinâmica de sobreposição variável ao longo do tempo (Figura 1b). Em quase todos os subdomínios do tema agrofloresta, a partir de 2014, foram observadas palavras-chave frequentes para mudanças climáticas e sequestro de carbono. No entanto, antes de 2014 o contexto foi diferente. Essa co-ocorrência permitiu visualizar possibilidades em atender diversos campos da ciência, sobretudo para os desafios impostos na produção sustentável, o que pode incluir as premissas existentes para o bem-estar único.

García-Pinillos et al. (2016) destacaram 10 temas sobre Bem-estar Único: 1- Redução do abuso de animais e humanos; 2- Melhor bem-estar animal - enfrentando problemas sociais; 3- Relações entre a melhoria do bem-estar animal e a segurança alimentar; 4- Melhor bem-estar animal melhor bem-estar humano; 5- Abordagens multidisciplinares mais eficientes; 6- Melhores chances de vida - reabilitação humana e realocação de animais; 7- Melhor bem-estar animal e do agricultor - melhoria da produtividade agrícola; 8- Melhor bem-estar animal - abordando a pobreza e o apoio da comunidade local; 9- Melhor bem-estar animal - melhoria da segurança alimentar e sustentabilidade; e 10- Aumento da biodiversidade - melhoria do bem-estar humano.

Varios autores descreveram que existe uma interconexão entre a agrofloresta com o meio ambiente e o bem-estar de animais e humanos (ETSHEKAPE et al., 2018), que pode ser observada com a interação humana e animal de estimação 
(DOS SANTOS et al., 2018) e animais de produção (BIRD et al., 1995; MANEVSKI et al., 2019; MASSACCESI et al., 2019); como também, indiretamente com animais selvagens (BENAVIDES et al., 2017; ROMERO-BAUTISTA et al., 2020). Além disso, a agrofloresta pode gerar serviços vinculados às dimensões biofísicas e ecossistêmicas de suporte a vida da terra (THOMPSON et al., 2011), e potencialmente no campo de saúde mental humana (BRATMAN et al., 2019; WHITE et al., 2019), que atualmente vem sendo pesquisado na ciência do bem-estar (BRYMER et al., 2019; PRITCHARD et al., 2020).

A interação existente entre humano e animais, bem como a interação entre animais domesticados e a vida selvagem que possibilitem desempenhar seus comportamentos naturais (DOS SANTOS et al., 2018), requer atenção. Há uma evolução para sistemas mais intensivos e mais controlados, nos quais a espécie humana e animal está inserida atualmente, no entanto, deve ser considerado que existem riscos a saúde humana e animal a serem monitorados, devido a possível inserção de espécies selvagens nesses ambientes, uma vez que, não há imediato controle sobre possíveis zoonoses que podem ser transmitidas, além de considerar o impacto da interação humana-animal sobre o comportamento natural destas espécies (MURRAY et al., 2016).

Há também preocupação com o desenvolvimento crescente de territórios agrícolas e pecuários extensivos e ou extrativistas, com o aumento de áreas urbanas e, demais práticas que podem levar a redução das áreas naturais. Esta perda de biodiversidade pode impulsionar o aparecimento de pragas, doenças e outros; como já destacado por De Oliveira Padilha et al. (2019) para malária; zoonoses por Murray et al. (2016) e arbovirus por Catenacci et al. (2018).

Os principais atores e partes interessadas veem pontos positivos na adoção da agrofloresta como melhoria da biodiversidade, habitats de vida selvagem, saúde, bem-estar animal, estética da paisagem (GARCÍA DE JALÓN et al., 2018), segurança alimentar, bem-estar social (FAHMI et al., 2018), além de gerar renda adicional (JESSY et al., 2017) um dos pontos para atingir o bem-estar econômico (RUGALEMA et al., 1994). Em contraste, apontaram que o aumento da mãode-obra, a complexidade do trabalho, os custos de gestão e os encargos administrativos, são vistos como os aspectos negativos mais importantes (GARCÍA DE JALÓN et al., 2018).

Há uma busca pelo equilíbrio e exploração sustentável dos recursos naturais; de modo que se possa atender a demanda de alimentos, o bem-estar humano e animal sem agredir a natureza. Sistemas agroflorestais podem trazer efeitos positivos diversos, sobretudo, pela combinação de valores de produção e biodiversidade, comparado a outros sistemas agrícolas e pecuários convencionais. Vale ressaltar que sistemas agroflorestais não substituem as florestas naturais, devido ao valor irreversível da biodiversidade
(SAGASTUY; KRAUSE, 2019); ainda segundo estes autores, as agroflorestas poderiam servir de um plano de conservação da biodiversidade.

A adoção de sistemas agroflorestais dependerá das características da região, econômicas e geográficas, acesso a informações, educação, atitudes e capital relacional (ZEWELD et al., 2018). Para atingi-los são necessárias tecnologias apropriadas, especialização na atividade, arranjos institucionais e políticas de apoio (BORREMANS et al., 2016).

\section{Conclusão}

Há forte potencial para avanço de pesquisas sobre o bemestar único dentro de sistemas agroflorestais. Estes podem ser intensificados por meio de conhecimentos multidisciplinares e pelas interconexões existentes. Há ainda, a necessidade de mais estudos que englobam humano-animal-natureza em sistemas agroflorestais.

\section{Referências}

BENAVIDES, C. et al. Home range and habitat use by pacas in a montane tropical forest in Bolivia. Acta Amaz., v.47, n.3, p.227236, 2017. doi: 10.1590/1809-4392201603163.

BIRD, P.R. et al. Animal production under a series of Pinus radiata-pasture agroforestry systems in South-West Victoria, Australia. Aust. J. Agric. Res., v.46, n.6, p.1299-1310, 1995. doi: 10.1071/AR9951299.

BORREMANS, L. et al. A sociopsychological analysis of agroforestry adoption in Flanders: understanding the discrepancy between conceptual opportunities and actual implementation. Agroecol. and Sustain. Food Syst., v.40, n.9, p.1008-1036, 2016. doi: 10.1080/21683565.2016.1204643.

BRATMAN, G.N. et al. Nature and mental health: An ecosystem service perspective. Sci. $A d v$. v.5, n.7, 2019. doi: 10.1126/sciadv. aax0903.

BROOM, D.M. Land and water usage in beef production systems. Anim., v.9, n.6, p.286, 2019. doi: 10.3390/ani9060286.

BROOM, D.M.; JOHNSON, K.G. One welfare, one health, one stress: humans and other animals. Stress Animal Welfare, v.19, p.1-13, 2019. doi: http://doi-org-443.webvpn.fjmu.edu. cn/10.1007/978-3-030-32153-6_1.

BRYMER, E. et al. One health: The wellbeing impacts of humannature relationships. Front. Psychol., v.10, p.1611, 2019. doi: 10.3389/fpsyg.2019.01611.

CARVALHO, P.C.D.F. et al. Definitions and terminologies for integrated crop-livestock system. Rev. Cienc. Agron., v.45, p.1040-1046, 2014. doi: 10.1590/S1806-66902014000500020.

CATENACCI, L.S. et al. Diversity patterns of hematophagous insects in Atlantic forest fragments and human-modified areas of southern Bahia, Brazil. J. Vector Ecol., v. 43, n. 2, 293-304, 2018. doi: $10.1111 /$ jvec. 12313 .

CÓRDOVA, R. et al. Mountain farming systems' exposure and sensitivity to climate change and variability: agroforestry and conventional agriculture systems compared in Ecuador's Indigenous Territory of Kayambi People. Sustain., v.11, n.9, p.2623, 2019. doi: 10.3390/su11092623.

COSTANZA, R. Toward an operational definition of health. In: COSTANZA, R.; NORTON, B.; HASKELL, B.D. Ecosystem 
health: new goals for environmental management. Washington: Inland Press, 1992. p.239-256. doi: 10.2307/2136932.

DE SOUZA, M.P. et al. Potential of microalgal bioproducts: general perspectives and main challenges. Waste Biomass Valorization, v.10, n.8, p.2139-2156, 2019. doi: 10.1007/s12649018-0253-6.

DE OLIVEIRA PADILHA, M.A. et al. Comparison of malaria incidence rates and socioeconomic-environmental factors between the states of Acre and Rondônia: a spatio-temporal modelling study. Malar. J., v.18, n.1, p.1-13, 2019. doi: 10.1186/ s12936-019-2938-0.

DOS SANTOS, C.L. et al. Human behaviors determine the direct and indirect impacts of free-ranging dogs on wildlife. $J$. Mammal., v.99, n.5, p.1261-1269, 2018. doi: 10.1093/jmammal/ gyy077.

DING, X.; YANG, Z. Knowledge mapping of platform research: a visual analysis using VOSviewer and CiteSpace. Electron. Commer. Res., p. 1-23, 2020. doi: 10.1007/s10660-020-09410-7.

ETSHEKAPE, P.G. et al. Tree planting in urban and peri-urban of Kinshasa: Survey of factors facilitating agroforestry adoption. Urban Forest. \& Urban Green., v.30, p.12-23, 2018. doi: 10.1016/j.ufug.2017.12.015.

FAHMI, M.K.M. et al. O. Impact of agroforestry parklands on crop yield and income generation: case study of rainfed farming in the semi-arid zone of Sudan. Agrofor. Syst., v.92, n.3, p.785800. 2018. doi: 10.1007/s10457-016-0048-3.

GARCÍA DE JALÓN, S. et al. How is agroforestry perceived in Europe? An assessment of positive and negative aspects by stakeholders. Agrofor. Syst., v. 92, n. 4, p. 829-848, 2018. doi: 10.1007/s10457-017-0116-3.

GARCÍA PINILLOS, G.R. et al. One welfare-a platform for improving human and animal welfare. Vet. Rec., v. 179, n. 16, p. 412-413, 2016. doi: 10.1136/vr.i5470.

GEORGE, S.J. et al. A sustainable agricultural landscape for Australia: a review of interlacing carbon sequestration, biodiversity and salinity management in agroforestry systems. Agric. Ecosyst. Environ., v. 163, p.28-36, 2012. doi: 10.1016/j. agee.2012.06.022.

HUMBOLDT-DACHROEDEN, S. et al. The state of one health research across disciplines and sectors-a bibliometric analysis. One Health, v. 10, p. 100146, 2020. doi: 10.1016/j. onehlt.2020.100146.

JESSY, M.D. et al. Possibilities of diverse rubber-based agroforestry systems for smallholdings in India. Agrofor. Syst., v. 91 , n. 3, p. 515-526, 2017. doi:10.1007/s10457-016-9953-8.

LASCO, R.D. et al. Agroforestry systems: helping smallholders adapt to climate risks while mitigating climate change. Wiley Interdisciplinary Reviews: Clim. Chang., v.5, n.6, p.825-833, 2014. doi: 10.1002/wcc.301.

LUNDMARK, F. et al. Intentions and values in animal welfare legislation and standards. J. Agric. Environ. Ethics, v. 27, n. 6, p. 991-1017, 2014. doi: 10.1007/s10806-014-9512-0.

MANEVSKI, K. et al. Effect of poplar trees on nitrogen and water balance in outdoor pig production-A case study in Denmark. Sci. Total Environ., v.646, p.1448-1458, 2019. doi: 10.1016/j. scitotenv.2018.07.376.

MBOW, C. et al. Agroforestry solutions to address food security and climate change challenges in Africa. Curr. Opin. in Environ. Sustain., v.6, p.61-67, 2014. doi: 10.1016/j.cosust.2013.10.014.

MASSACCESI, L. et al. Geese reared in vineyard: soil, grass and animals interaction. Anim., v.9, n.4, p.179, 2019. doi: 10.3390/ ani9040179.

MURRAY, K.A. et al. Emerging viral zoonoses from wildlife associated with animal-based food systems: risks and opportunities. In: Food safety risks from wildlife. Springer, Cham. p.31-57, 2016. doi: 10.1007/978-3-31924442-6_2.

NAIR, P.K.R. An introduction to agroforestry. Dordrecht: ICRAF. 1993.

NAIR, P.K.R. Agroforestry systems and environmental quality: introduction. J. Environ. Qual., v.40, n.3, p.784-790, 2011. doi: 10.2134/jeq2011.0076. PMID: 21546663.

PRITCHARD, A. et al. The relationship between nature connectedness and eudaimonic well-being: A meta-analysis. $J$. Happiness Stud., v.21, n.3, p.1145-1167, 2020. doi: 10.1007/ s10902-019-00118-6.

RAPPORT, D.J. et al. Assessing ecosystem health. Trends in Ecol. and Evol., v.13, n.10, p.397-402, 1998. doi: 10.1016/ S0169-5347(98)01449-9.

ROMERO-BAUTISTA, Y.A. et al. Environmental interactions between people and birds in semiarid lands of the Zapotitlán Valley, Central Mexico. J. Ethnobiol. Ethnomed., v.16, p.1-14, 2020. doi: 10.1186/s13002-020-00385-1.

ROSENSTOCK, T.S. et al. A planetary health perspective on agroforestry in Sub-Saharan Africa. One Earth, v. 1, n. 3, p. 330344, 2019.

doi: 10.1016/j.oneear.2019.10.017.

RUGALEMA, G.H. et al. The homegarden agroforestry system of Bukoba district, North-Western Tanzania. 3. An economic appraisal of possible solutions to falling productivity. Agrofor. Syst., v. 28, n. 3, p. 227-236, 1994. https://doi.o10.1007/ BF00704758.

SAGASTUY, M., KRAUSE, T. Agroforestry as a biodiversity conservation tool in the atlantic forest? motivations and limitations for small-scale farmers to implement agroforestry systems in North-Eastern Brazil. Sustain., v.11, n.24, p. 6932, 2019. doi: $10.3390 /$ su11246932.

TARAZONA, A.M. et al. Human relationships with domestic and other animals: one health, one welfare, one biology. Anim., v.10, n.1, p.43, 2020. doi: 10.3390/ani10010043.

THIERMANN, A.B.; BABCOCK, S. Animal welfare and international trade. Rev. Sci. Tech.- Int. Epizoot., v.24, n.2, p.747, 2005. doi: 10.20506/RST.24.2.1600.

THOMPSON, I.D. et al. Forest biodiversity and the delivery of ecosystem goods and services: translating science into policy. BioSci., v.61, n.12, p.972-981, 2011. doi: 10.1525/ bio.2011.61.12.7.

THORENZ, A. et al. Assessment of agroforestry residue potentials for the bioeconomy in the European Union. J. Cleaner Prod., v.176, p.348-359, 2018. doi: 10.1016/j.jclepro.2017.12.143.

TORRES, C.M.M.E. et al. Greenhouse gas emissions and carbon sequestration by agroforestry systems in southeastern Brazil. Sci. Rep., v.7, n.1, p.1-7, 2017. doi: 10.1038/s41598-017-16821-4.

WHITE, M.P. et al. Spending at least 120 minutes a week in nature is associated with good health and wellbeing. Sci. Rep., v.9, n.1, p.1-11, 2019. doi: 10.1038/s41598-019-44097-3.

ZERMEÑO-HERNÁNDEZ, I. et al. Agricultural land-use diversity and forest regeneration potential in human-modified tropical landscapes. Agric. Ecosyst. Environ., v.230, p.210-220, 2016. doi: 10.1016/j.agee.2016.06.007. 
ZEWELD, W. et al. Impacts of socio-psychological factors on actual adoption of sustainable land management practices in dryland and water stressed areas. Sustain., v.10, n.9, p.2963, 2018. doi: 10.3390/su10092963.
ZOMER, R.J. et al. Global tree cover and biomass carbon on agricultural land: the contribution of agroforestry to global and national carbon budgets. Sci. Rep., v.6, n.1, p 1-12, 2016. doi: 10.1038/srep29987. 